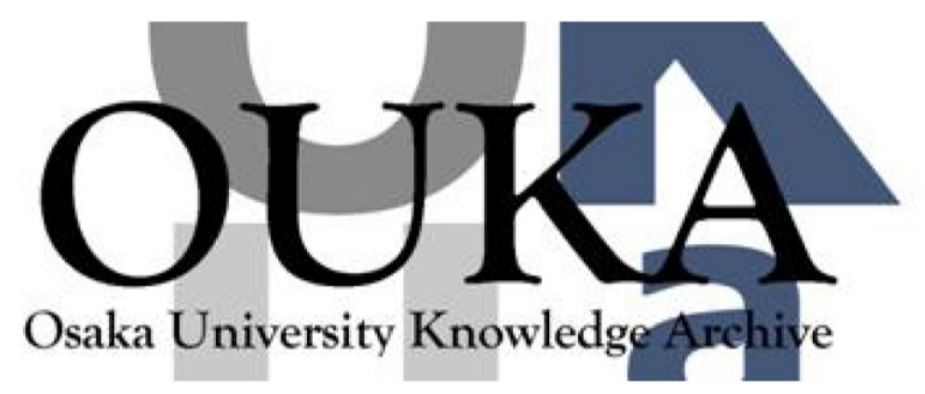

\begin{tabular}{|c|l|}
\hline Title & $\begin{array}{l}\text { Rigid helical conformation of Curdlan } \\
\text { tris(phenylcarbamate) in solution }\end{array}$ \\
\hline Author(s) & $\begin{array}{l}\text { Ochiai, Takaaki; Nakamura, Yasuko; Yoshikawa, } \\
\text { Chiaki et al. }\end{array}$ \\
\hline Citation & Polymer. 53(18) p. 3649-p. 3950 \\
\hline Issue Date & $2012-08-17$ \\
\hline oaire:version & AM \\
\hline URL & https://hdl. handle. net/11094/25934 \\
\hline rights & \\
\hline Note & \\
\hline
\end{tabular}

Osaka University Knowledge Archive : OUKA

https://ir. Library. osaka-u. ac. jp/

Osaka University 


\section{Rigid helical conformation of curdlan tris(phenylcarbamate) in solution}

Takaaki Ochiai, Ken Terao *, Yasuko Nakamura, Chiaki Yoshikawa, Takahiro Sato

Department of Macromolecular Science, Graduate School of Science, Osaka University, 1-1 Machikaneyama-cho, Toyonaka, Osaka 560-0043, Japan

* Corresponding author. Tel. +81 66850 5459; fax: +81 668505461.

E-mail address: kterao@chem.sci.osaka-u.ac.jp (K. Terao).

Keywords: Polysaccharide derivative; hydrogen bond; chain stiffness 


\section{ABSTRACT}

Light and small-angle X-ray scattering and viscosity measurements were made for four curdlan tris(phenylcarbamate) (CdTPC) samples having quite narrow dispersity indices (DI) and ranging in the molecular weight between $6 \times 10^{4}$ and $2 \times 10^{6}$, to determine weightaverage molecular weights $M_{\mathrm{w}}$, radii of gyration $<S^{2}>$, particle scattering functions $P(q)$, and intrinsic viscosities $[\eta]$ in tetrahydrofuran (THF) and 4-methyl-2-pentanone (MIBK). Size exclusion chromatography equipped with light scattering and/or viscosity detectors was performed for some broad DI samples in THF also to determine $\left\langle S^{2}\right\rangle$ and [ $\eta$ ] as a function of $M_{\mathrm{w}}$. The solution data were analyzed in terms of the wormlike-chain model to estimate the Kuhn segment length of $57 \mathrm{~nm}$ in THF and $17 \mathrm{~nm}$ in MIBK and the helix pitch per residue $h$ of $0.39 \mathrm{~nm}$ in THF and $0.51 \mathrm{~nm}$ in MIBK. Since the former $h$ value is almost equivalent to that for single helical curdlan and its acetate derivative in crystal, it is reasonably suggested that the local conformation of CdTPC in THF is close to the tightly wound helix stabilized by the intramolecular hydrogen bonding, which was detected from the solution IR spectra. 


\section{Introduction}

Cellulose ( $\beta$-1,4-glucan), amylose ( $\alpha$-1,4-glucan), and their phenylcarbamate derivatives have been widely investigated on their solution properties since 1960's [1]. Because the derivatives have three $\mathrm{C}=\mathrm{O}$ and $\mathrm{NH}$ groups on each repeat unit, intramolecular hydrogen bonding (H-bonding) plays an important role on the conformation in solution [2]. Indeed, amylose tris(phenylcarbamate) (ATPC) has significantly higher chain stiffness than that for amylose in water and dimethylsulfoxide [1,3] when the solution data were analyzed in terms of the Kratky-Porod wormlike chain model [4]. The H-bonds are observable in the solution infrared (IR) absorption spectra as the split amide I band [5,6]. We recently found that the H-bonds significantly stiffen amylose tris(n-butylcarbamate) (ATBC) in tetrahydrofuran (THF) and the chain stiffness parameter $\lambda^{-1}$ (the Kuhn segment length) is the highest (= 75 $\mathrm{nm})$ in all the known polysaccharide carbamates whereas ATBC in methanol $(\mathrm{MeOH})$ has loosely and rather flexible main chain $\left(\lambda^{-1}=11 \mathrm{~nm}\right)$ [7]. This implies that other rodlike carbamate derivatives could be found if we select an appropriate main chain and side groups. Polysaccharide derivatives having stiff main chain are of still interest since such polymer may have lyotropic liquid crystallinity. In actuality, liquid crystallinity of cellulose and chitosan phenylcarbamate was recently reported [8,9]. Since the phase boundary concentration decreases remarkably with increasing chain stiffness, we might find liquid crystalline phase even at lower concentrations if we find a stiffer polysaccharide derivative.

As an attempt, we chose curdlan tris(phenylcarbamate) (CdTPC) of which chemical structure is shown in Fig. 1. Curdlan is a (1,3)- $\beta$-D-glucan produced by a bacteria and having triple helical structure in the native state $[10,11]$. Interestingly, the intrinsic viscosities $[\eta]$ of an unfractionated CdTPC sample depend significantly on the solvent as shown in Table 1 and the highest $[\eta]$ was found in THF as is the case of ATBC [12]. This solvent dependence is much different from phenylcarbamate derivatives of cellulose and amylose [13,14]: [ $\eta]$ for them in THF or 1,4-dioxane are not much larger than those in ketones and esters. This 
suggests that CdTPC may have rigid helical conformation in THF. Although tris carbamate derivatives of curdlan were synthesized for a chiral stationary phase [15], their solution properties have not been reported yet. Dimensional and hydrodynamic properties of CdTPC in THF and 4-methyl-2-pentanone (MIBK) in which CdTPC would behave as rather flexible chain were therefore analyzed in terms of the wormlike chain model mainly to determine $\lambda^{-1}$ and the helix per residue. Furthermore, the infrared absorption spectrum in THF was determined to compare H-bonding feature with that for ATPC and cellulose tris(phenylcarbamate) (CTPC).

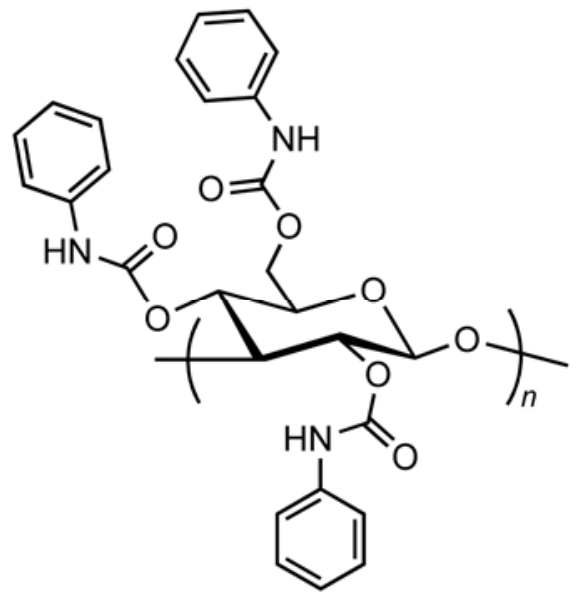

Fig. 1. Chemical structure of CdTPC. 
Table 1. Intrinsic viscosities for an unfractionated CdTPC sample at $25^{\circ} \mathrm{C}$.

\begin{tabular}{cc}
\hline solvent & {$[\eta]\left(\mathrm{cm}^{3} \mathrm{~g}^{-1}\right)$} \\
\hline THF & 1500 \\
cyclohexanone & 1200 \\
2-butanone & 1170 \\
methyl acetate & 890 \\
MIBK & 805 \\
L-ethyl lactate & 788 \\
DL-ethyl lactate & 748 \\
isopentyl acetate & 700 \\
2,4-dimethyl-3-pentanone & 687
\end{tabular}

\section{Experimental}

\subsection{Samples and Solvents}

CdTPC was synthesized from a commercially available curdlan sample (Wako). It (3.0 g) was dried in vacuum at $90{ }^{\circ} \mathrm{C}$ for 2 hours and dispersed in dry pyridine under nitrogen atmosphere. An excess amount (20 g) of phenylisocyanate was added to the suspension and the resultant reaction mixture was gently stirred at $120{ }^{\circ} \mathrm{C}$ for $15-20$ hours. The obtained clear brown solution was diluted with acetone to reduce the viscosity, precipitated into $\mathrm{MeOH}$, and washed with $\mathrm{MeOH}$ several times, to obtain white flocculent sample. It should be noted that this polymer is soluble neither in $\mathrm{MeOH}$ nor water. This CdTPC sample was dried in vacuum overnight (CdTPC-N). To prepare lower molecular weight CdTPC samples, ultrasonic waves $(20 \mathrm{kHz}$ ) were irradiated to $D L$-ethyl lactate solution of CdTPC-N by using a Branson Sonifier 450 Advanced for more than 30 hours. The resultant samples were reprecipitated into methanol (CdTPC-S and CdTPC-M). Successive fractional precipitation 
was performed for these samples with acetone as a solvent and methanol as a precipitant. Four appropriate middle fractions, CdTPC60K, CdTPC80K, CdTPC140K, and CdTPC1500K, as well as CdTPC-N, CdTPC-S, and CdTPC-M were chosen for this study. The degree of substitution was determined to be $3.0 \pm 0.2$ for all these samples from the mass ratio of carbon to nitrogen measured by elemental analysis. The chemical structure was also confirmed by using ${ }^{1} \mathrm{H}-\mathrm{NMR}$ in $\mathrm{CDCl}_{3}$ and FT-IR (KBr method). The dispersity index (DI, the ratio of weight- to number-average molecular weights) was estimated to be $1.02-1.10$ for CdTPC60K, CdTPC80K, CdTPC140K, and CdTPC1500K and ca. 1.3 for CdTPC-N, CdTPC-S, and CdTPC-M by size-exclusion chromatography (SEC) combined with low- and right-angle laser light scattering and refractive index detectors.

\subsection{Static Light Scattering (SLS) and SEC equipped with Multi-angle Light Scattering} (MALS) Detector.

SLS measurements were carried out for CdTPC60K, CdTPC80K, CdTPC140K, and CdTPC1500K in THF and CdTPC140K and CdTPC1500K in MIBK all at $25{ }^{\circ} \mathrm{C}$ on a Fica50 light scattering photometer with vertically polarized incident light of $436 \mathrm{~nm}$ wavelength $\left(\lambda_{0}\right)$. Four solutions with different concentration were measured for each sample. Experimental details were described in ref. [5]. SEC-MALS measurements were made for the three polydisperse samples, CdTPC-N, CdTPC-S, and CdTPC-M on a Wyatt Dawn DSP photometer $\left(\lambda_{0}=633 \mathrm{~nm}\right)$ and a refractive index detector equipped with two SEC columns (Shodex Multipore-HxLM) in THF at $25{ }^{\circ} \mathrm{C}$. Flow rate, volume of the injection loop, and the polymer mass concentration $c$ were set to be $1.0 \mathrm{~mL} / \mathrm{min}, 100 \mu \mathrm{L}$, and $1 \times 10^{-3} \mathrm{~g} \mathrm{~cm}^{-3}$, respectively. The obtained data from SLS and SEC-MALS were analyzed in terms of the Berry square-root plot [16] to determine the weight-average molecular weight $M_{\mathrm{w}}$, the $z$ average mean square radius of gyration $\left\langle S^{2}\right\rangle_{\mathrm{z}}$, and the second virial coefficient $A_{2}$, which is in the order of $10^{-4} \mathrm{~mol} \mathrm{~g}^{-2} \mathrm{~cm}^{3}$ both in THF and MIBK; hence they are good solvents for 
CdTPC. It should be noted that the obtained $M_{\mathrm{w}}$ in the two solvents are almost equivalent (< $\pm 2 \%)$ to each other. The specific refractive index increment $\partial n / \partial c$ for CdTPC80K in THF and MIBK at $25{ }^{\circ} \mathrm{C}$ was determined by using an Otsuka differential refractometer DRM-120. The $\partial \mathrm{n} / \partial c$ values at $\lambda_{0}=436 \mathrm{~nm}$ were determined to be $0.215 \mathrm{~cm}^{3} \mathrm{~g}^{-1}$ and $0.190 \mathrm{~cm}^{3} \mathrm{~g}^{-1}$ in THF and MIBK, respectively.

\subsection{Small-Angle $X$-Ray Scattering (SAXS)}

SAXS measurements were made with an imaging plate detector at the BL40B2 beamline at SPring-8 for CdTPC60K in THF and MIBK at $25{ }^{\circ} \mathrm{C}$ (see ref [5] for experimental details). The camera length and the wavelength were set to be $1500 \mathrm{~mm}$ and $0.1 \mathrm{~nm}$, respectively. The excess scattering intensities measured for four different concentrations were analyzed by means of the Berry square-root plot to determine $\left\langle S^{2}\right\rangle_{z}$ and the particle scattering function $P(q)$, where $q$ denotes the magnitude of the scattering vector.

\subsection{Viscometry}

Viscosity measurements were made using a four-bulb low-share capillary viscometer of the Ubbelohde type for CdTPC1500K and a conventional capillary viscometer for CdTPC60K, CdTPC80K, and CdTPC140K to determine $[\eta]$ and the Huggins constant in THF and MIBK at $25{ }^{\circ} \mathrm{C}$. The Huggins constants were obtained to be $0.3-0.5$. Intrinsic viscosities in THF were also determined as a function of $M_{\mathrm{w}}$ for CdTPC-N and CdTPC-M (broad DI samples) by using SEC equipped with TriSEC302TDA (Viscotek) including viscosity, two-angle light scattering, and refractive index detectors.

\subsection{Infrared Absorption}

IR spectra for CdTPC1500K and a CTPC sample [14] $\left(M_{\mathrm{w}}=2.62 \times 10^{6}\right)$ in THF at $25^{\circ} \mathrm{C}$ were determined using a Bio-Rad FTS-300 Fourier transform infrared spectrometer with a 
solution cell made of $\mathrm{CaF}_{2}$ (0.05 mm path length). Concentrations of the test solutions were set to be $1.0 \times 10^{-2} \mathrm{~g} \mathrm{~cm}^{-3}$.

\section{Results and Discussion}

\subsection{Dimensional and Hydrodynamic Properties}

Figs. 2 and 3 show the particle scattering function $P(q)$ obtained by SLS and SAXS for the CdTPC samples in THF and MIBK at $25{ }^{\circ} \mathrm{C}$, in the forms of the Berry plots $\left[P(q)^{-1 / 2}\right.$ vs $q^{2}$; Fig. 2] and of the the Holtzer plots $[q P(q)$ vs $q$; Fig. 3]. Dashed lines in Fig. 2 indicate the initial slope to determine $\left\langle S^{2}\right\rangle_{\mathrm{z}}$. The resultant $M_{\mathrm{w}}$ and $\left\langle S^{2}\right\rangle_{\mathrm{z}}$ obtained by SLS and SAXS, as well as $[\eta]$, are summarized in Table 2.
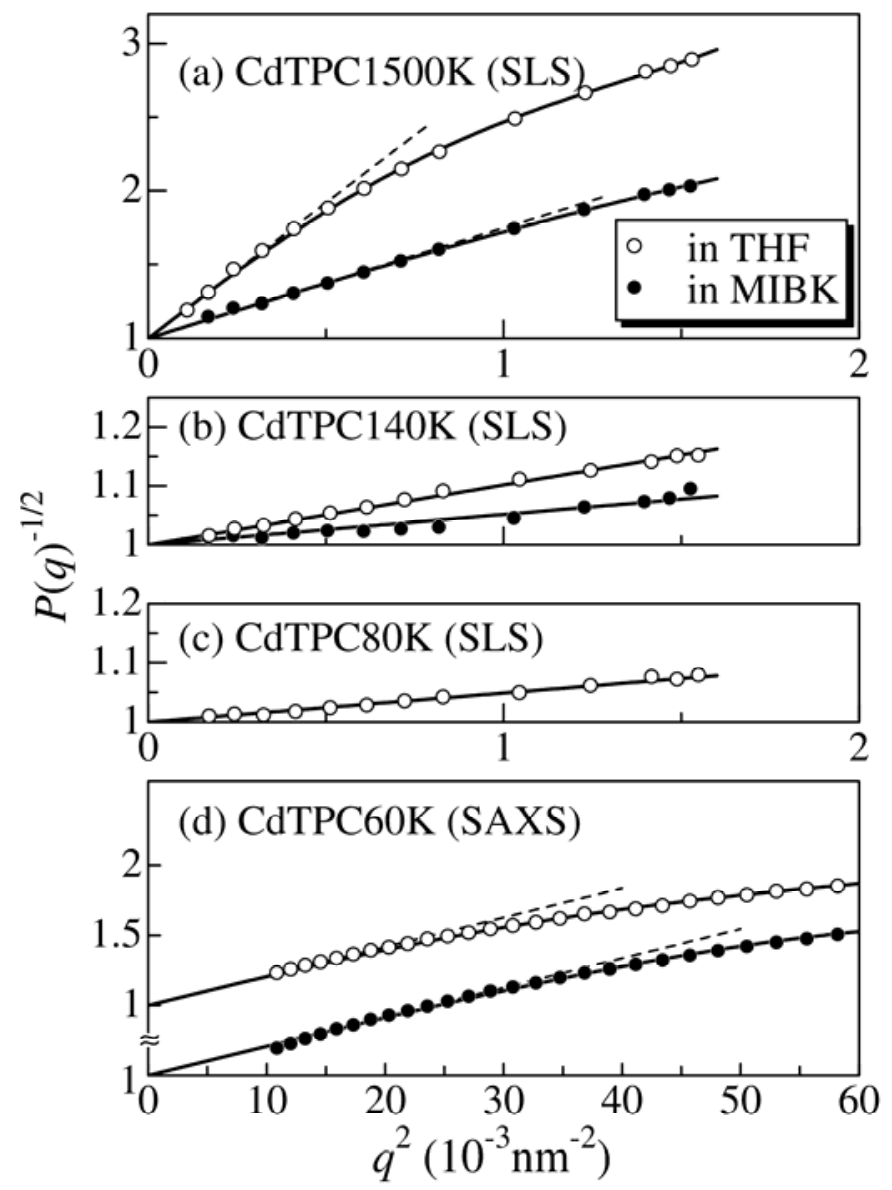

Fig. 2. Berry plots for CdTPC samples in THF (unfilled circles) and in MIBK (filled circles) at $25^{\circ} \mathrm{C}$. (a) For CdTPC1500K (SLS). (b) For CdTPC140K (SLS). (c) For CdTPC80K (SLS). (d) For CdTPC60K (SAXS). 


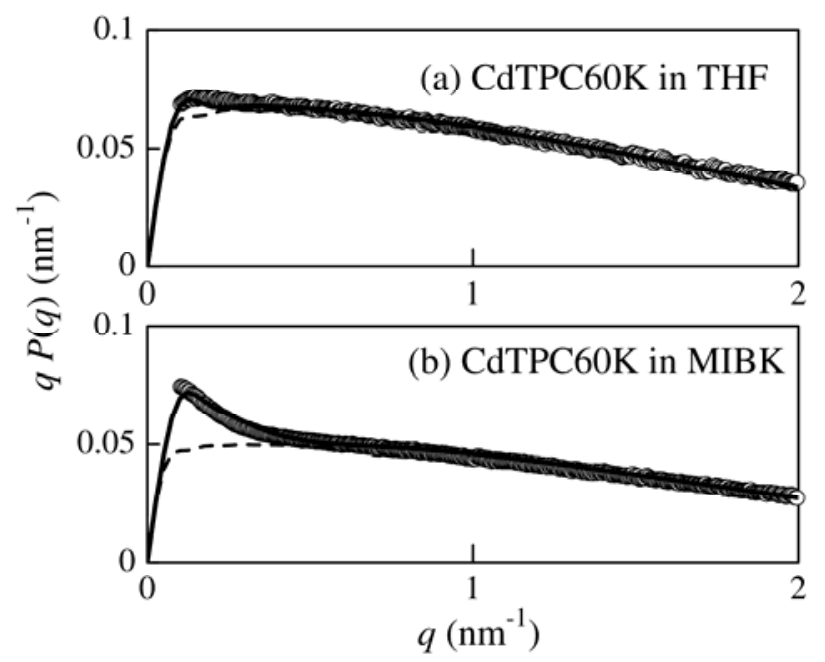

Fig. 3. Holtzer plots for CdTPC60K in THF (a) and MIBK (b) at $25{ }^{\circ} \mathrm{C}$. Solid curves, theoretical values for the unperturbed wormlike cylinders with the parameters in Table 3. Dashed curves, theoretical values in the rod limit $\left(\lambda^{-1}=\infty\right)$.

Table 2. Molecular characteristics of CdTPC in THF and MIBK at $25^{\circ} \mathrm{C}$.

\begin{tabular}{|c|c|c|c|c|c|}
\hline \multirow{2}{*}{ Sample } & \multirow{2}{*}{$M_{\mathrm{w}} / 10^{4}$} & \multicolumn{2}{|c|}{ in $\mathrm{THF}$} & \multicolumn{2}{|c|}{ in MIBK } \\
\hline & & $\left\langle S^{2}>_{\mathrm{z}}^{1 / 2}(\mathrm{~nm})\right.$ & {$[\eta]\left(\mathrm{cm}^{3} \mathrm{~g}^{-1}\right)$} & $\left\langle S^{2}>_{\mathrm{z}}^{1 / 2}(\mathrm{~nm})\right.$ & {$[\eta]\left(\mathrm{cm}^{3} \mathrm{~g}^{-1}\right)$} \\
\hline CdTPC60K & $6.08^{\mathrm{a}}$ & $11.7^{b}$ & 56 & $11.0^{b}$ & 46 \\
\hline CdTPC80K & $8.27^{\mathrm{a}}$ & $17.1^{\mathrm{a}}$ & 61 & & 65 \\
\hline CdTPC140K & $14.1^{\mathrm{a}}$ & $24.7^{\mathrm{a}}$ & 113 & $17.6^{\mathrm{a}}$ & 80 \\
\hline CdTPC1500K & $148^{\mathrm{a}}$ & $105^{\mathrm{a}}$ & 1340 & $67^{\mathrm{a}}$ & 690 \\
\hline
\end{tabular}

${ }^{\mathrm{a}}$ SLS. ${ }^{\mathrm{b}}$ SAXS.

Fig. 4 displays the plots of $\left\langle S^{2}\right\rangle_{\mathrm{z}}$ against the weight-average degree of polymerization $N_{\mathrm{w}}$ for CdTPC in the two solvents and for curdlan in $0.3 \mathrm{M}$ aqueous $\mathrm{NaOH}$ [17] and in diluted cadoxen [18]. The gyration radii of CdTPC are much larger than those for curdlan, indicating CdTPC has much more extended conformation than curdlan in solution. Furthermore, CdTPC in THF has larger $\left\langle S^{2}\right\rangle_{\mathrm{z}}$ than that in MIBK and the Holtzer plots (Fig. 3) for CdTPC60K in THF has almost flat plateau around $q=0.2 \mathrm{~nm}^{-1}$ whereas that in MIBK has an appreciable peak, indicating significantly higher chain stiffness of CdTPC in THF than 
that in MIBK. These tendencies are more clearly recognizable in the plot of $[\eta] M_{0}$ against $N_{\mathrm{w}}$ as shown in Fig. 5, where $M_{0}$ is the molar mass of the repeat unit. It should be noted that we use [ $\eta] M_{0}$ and $N_{\mathrm{w}}$ instead of $[\eta]$ and $M_{\mathrm{w}}$ to correct the difference in the mass of repeat unit. These features of the solution data for CdTPC are typical for semiflexible polymers and therefore the data are analyzed in terms of the wormlike chain.

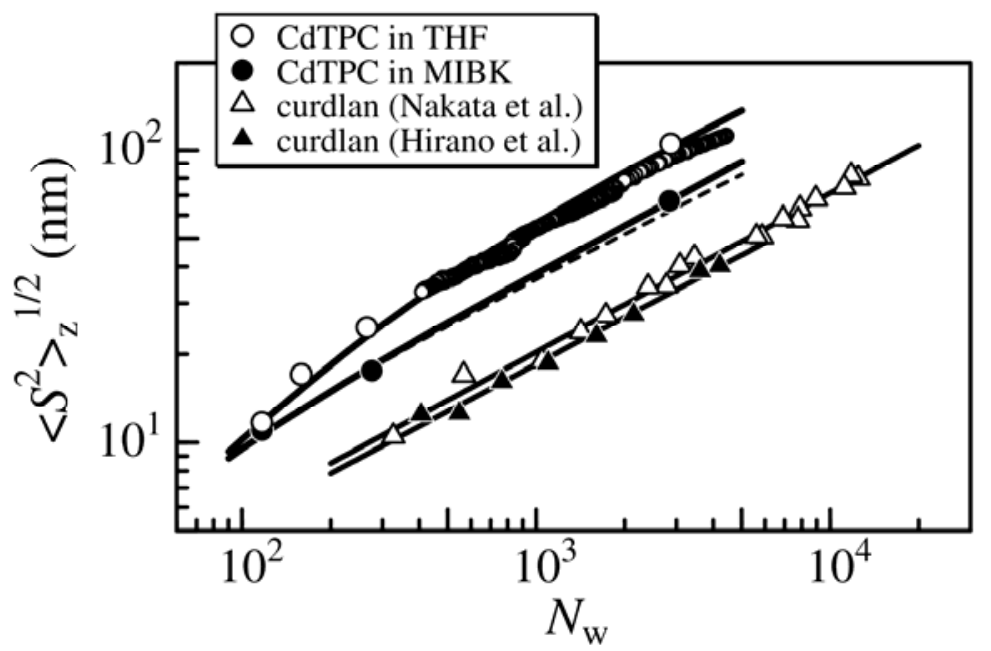

Fig. 4. $N_{\mathrm{w}}$-dependence of $\left\langle S^{2}\right\rangle_{\mathrm{z}}{ }^{1 / 2}$ for CdTPC in THF (unfilled circles) and in MIBK (filled circles) all at $25^{\circ} \mathrm{C}$. Open and filled triangles represent literature values for curdlan in $0.3 \mathrm{M}$ aqueous $\mathrm{NaOH}$ [17] and in water diluted cadoxen (1:1) [18]. Solid curves show theoretical values calculated for the wormlike chains with the parameters in Table 3 . The dashed curve shows the theoretical values for $B=0$. 


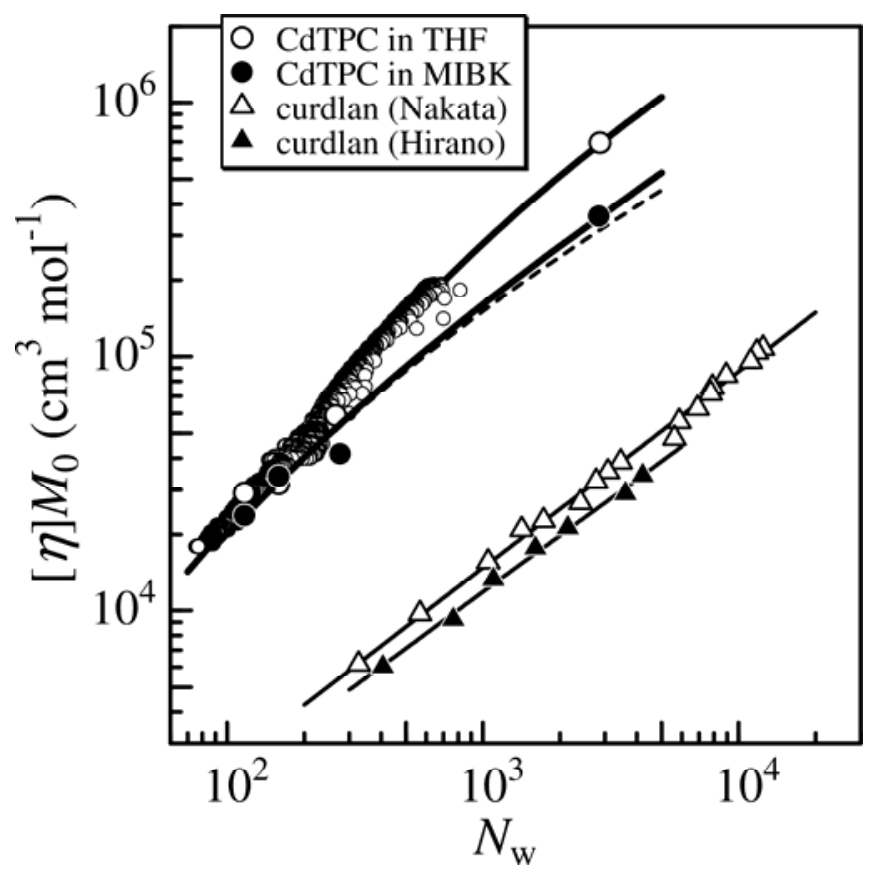

Fig. 5. $N_{\mathrm{w}}$-dependence of [ $\left.\eta\right] M_{0}$ for CdTPC in THF (unfilled circles) and in MIBK (filled circles) all at $25^{\circ} \mathrm{C}$. Unfilled and filled triangles represent literature values for curdlan in 0.3 M [17] aqueous $\mathrm{NaOH}$ and in water diluted cadoxen (1:1) [18]. Solid curves, the theoretical values calculated for the wormlike chains with the parameters in Table 3; dashed curve, theoretical values for $B=0$.

The theoretical radius of gyration $\left\langle S^{2}>\right.$ for the wormlike chain is obtained as the product of the unperturbed gyration radius $\left\langle S^{2}\right\rangle_{0}$ calculated by the Benoit-Doty equation [19]

$$
\left\langle S^{2}\right\rangle_{0}=\frac{M}{6 \lambda M_{\mathrm{L}}}-\frac{1}{4 \lambda^{2}}+\frac{M_{\mathrm{L}}}{4 \lambda^{3} M}-\frac{M_{\mathrm{L}}^{2}}{8 \lambda^{4} M^{2}}\left[1-\left.\exp \left(-\frac{2 \lambda M}{M_{\mathrm{L}}}\right)\right|_{]}\right.
$$

and the expansion factor estimated by the Domb-Barrett equation [20] in the quasi-twoparameter (QTP) theory $[21,22,23]$. Thus, $<S^{2}>$ is calculated from the molar mass per unit contour length $M_{\mathrm{L}}$, chain stiffness parameter $\lambda^{-1}$ (the Kuhn segment length, which corresponds to twice the persistence length), and the excluded-volume strength $B$. On the other hand, the particle scattering function $P(q)$ for the cylindrical wormlike chain is formulated by Nakamura and Norisuye [24] as functions of $M_{\mathrm{L}}, \lambda^{-1}$, and the chain diameter $d$. 
The excluded volume effect is not important in $P(q)$ at high $q$. In THF, $M_{\mathrm{L}}$ and $\lambda^{-1}$ are unequivocally determined from $\left\langle S^{2}>\right.$ data, and the excluded-volume effects on $\left\langle S^{2}>\right.$ are negligible even for the highest $M_{\mathrm{w}}$ sample even in MIBK, when assuming the $M_{\mathrm{L}}$ value from $P(q)$, the rest parameters are uniquely determined. This is most likely due to the quite high chain stiffness of CdTPC in MIBK comparing with typical flexible polymers $\left(\lambda^{-1} \sim 2 \mathrm{~nm}\right)$. On the other hand, from the curve fitting of the $P(q)$ data in Fig. $3, M_{\mathrm{L}}, \lambda^{-1}$, and $d$ in MIBK and $M_{\mathrm{L}}$ and $d$ in THF were determined. However, the chain stiffness in THF cannot be determined since CdTPC is too stiff in the solvent, and indeed, the obtained theoretical $P(q)$ (solid curve) in THF is closely fitted by that for the rod limit (dashed curve). Theoretical $<S^{2}>$ and $P(q)$ values calculated from the obtained parameters (see Table 3) reproduce the experimental data almost quantitatively as shown by solid curves in Figs. 3 and 4, respectively. The excluded-volume effects in MIBK seen as the discrepancy between solid and dashed curves in Fig. 3 are not significant even for the highest molecular weight sample. It should be noted that the chain thickness effect on $\left\langle S^{2}>\right.$ is negligible $(<0.3 \%$ ) for the investigated $M_{\mathrm{w}}$ range if we estimate the mean-square radius of gyration for the cylindrical wormlike chain by $<S^{2}>+d^{2} / 8$ [25]. 
Table 3. Wormlike chain parameters for CdTPC in THF and MIBK at $25^{\circ} \mathrm{C}$.

\begin{tabular}{ccccc}
\hline $\begin{array}{c}\text { CdTPC in THF } \\
\text { Method }\end{array}$ & $M_{\mathrm{L}}\left(\mathrm{nm}^{-1}\right)$ & $\lambda^{-1}(\mathrm{~nm})$ & $d(\mathrm{~nm})$ & \\
\hline$P(q)$ & $1360 \pm 50$ & $57^{\mathrm{a}}$ & $1.7 \pm 0.2$ & \\
$<S^{2}>_{\mathrm{z}}$ & $1290 \pm 50$ & $58 \pm 5$ & - & \\
{$[\eta]$} & $1360^{\mathrm{a}}$ & $56 \pm 5$ & $2.4 \pm 0.5$ & \\
\hline CdTPC in MIBK & & & $d(\mathrm{~nm})$ & $B(\mathrm{~nm})$ \\
Method & $M_{\mathrm{L}}\left(\mathrm{nm}^{-1}\right)$ & $\lambda^{-1}(\mathrm{~nm})$ & $1.6 \pm 0.2$ & - \\
\hline$P(q)$ & $1010 \pm 50$ & 17 & - & $1.0 \pm 0.5$ \\
$<S^{2}>_{\mathrm{z}}$ & $1010^{\mathrm{a}}$ & $16 \pm 2$ & $1.3 \pm 0.4$ & $1.0 \pm 0.5$ \\
{$[\eta]$} & $1010^{\mathrm{a}}$ & $19 \pm 2$ & & \\
\hline
\end{tabular}

${ }^{\mathrm{a}}$ Assumed.

Molecular-weight dependence of $[\eta]$ was analyzed in terms of the combination of the Yamakawa-Fujii-Yoshizaki theory $[26,27]$ for the unperturbed viscosity and the Barrett function [28] for the viscosity-expansion factor in the QTP scheme $[21,22,23]$. Thus, the four parameters, $M_{\mathrm{L}}, \lambda^{-1}, d$, and $B$ are needed to calculate the theoretical [ $\eta$ ]. Since these parameters cannot be determined unequivocally only from the $[\eta]$ data, we assumed the $M_{\mathrm{L}}$ value from $P(q)$ and the rest three parameters were determined to fit the data in Fig. 5; as in the case of $\left\langle S^{2}>\right.$, the excluded-volume effect in THF is negligibly small and that in MIBK is not significant.

The obtained parameters from the three methods listed in Table 3 are consistent with each other, suggesting that conformation of CdTPC in the two solvents can be modeled by the wormlike chain and the accurate parameters were obtained. Thus, the mean helix pitch (or contour length) per residue $h\left(=M_{0} / M_{\mathrm{L}}\right)$ and $\lambda^{-1}$ values are summarized in Table 4 along with those for ATPC and CTPC [14]. Interestingly, although the three polysaccharide phenylcarbamates have almost the same repeat unit, $\lambda^{-1}$ for CdTPC in THF is much larger 
than that for CTPC in THF and ATPC in 1,4-dioxane (DIOX) and it is rather close to that for the stiffest polysaccharide derivatives, that is, amylose tris(n-butylcarbamate) in THF $\left(\lambda^{-1}=\right.$ $75 \mathrm{~nm})$ [7] and amylose tris(3,5-dimetylphenylcarbamate) in MIBK ( $\left.\lambda^{-1}=73 \mathrm{~nm}\right)$ [29]. Indeed, CdTPC140K in THF $\left(c=0.48 \mathrm{~g} \mathrm{~cm}^{-3}\right)$ shows cholesteric liquid crystalline. Furthermore, $h$ for CdTPC in THF is close to that for single helical crystal of curdlan [30] and curdlan acetate [31], indicating that the CdTPC main chain has tightly wound single helical structure. On the one hand, CdTPC in MIBK has locally extended but rather flexible coil-like main chain, suggesting that the intramolecular H-bonds in MIBK should be less than that in THF and furthermore the H-bonding solvent molecules may extend the main chain of CdTPC as seen for ATPC [13].

Table 4. Values of helix pitch per residue $h$ and the Kuhn segment length $\lambda^{-1}$ for CdTPC, ATPC, and CTPC at $25^{\circ} \mathrm{C}$.

\begin{tabular}{cccc}
\hline Sample & Solvent & $h(\mathrm{~nm})$ & $\lambda^{-1}(\mathrm{~nm})$ \\
\hline CdTPC & THF & $0.39 \pm 0.02$ & $57 \pm 5$ \\
CdTPC & MIBK & $0.51 \pm 0.03$ & $17 \pm 2$ \\
ATPC $^{\text {a }}$ & DIOX & $0.34 \pm 0.01$ & $22 \pm 2$ \\
ATPC $^{\text {b }}$ & MIBK & $0.42 \pm 0.02$ & $24 \pm 2$ \\
CTPC $^{\text {c }}$ & THF & $0.49 \pm 0.04$ & 21 \\
\hline
\end{tabular}

${ }^{\mathrm{a}}$ Ref [5], ${ }^{\mathrm{b}}$ ref [13], ${ }^{\mathrm{c}}$ ref [14].

\subsection{Intramolecular H-bonding}

The above mentioned high chain stiffness as well as relatively small $h$ for CdTPC in THF should be something to do with the intramolecular H-bonding. Fig. 6 displays the IR spectra for CdTPC in THF around the amide I and II bands along with those for ATPC in DIOX [5] and CTPC in THF. The peak at $1754 \mathrm{~cm}^{-1}$ for ATPC was assigned for the 'free' $\mathrm{C}=\mathrm{O}$ groups and that at $1706 \mathrm{~cm}^{-1}$ is for $\mathrm{H}$-bonding $\mathrm{C}=\mathrm{O}$ groups [5,32]. Intramolecular $\mathrm{H}$-bonds are 
fewer for CTPC and the peak shifts to slightly higher wavenumber; this may reflect CTPC has fewer and weaker H-bonds comparing with ATPC. On the other hand, amide I band for CdTPC is obtained to be a broad single peak at $1735 \mathrm{~cm}^{-1}$, assigning weakly $\mathrm{H}$-bonding $\mathrm{C}=\mathrm{O}$ groups. In other words, CdTPC has weak but much larger number of intramolecular Hbonds in THF and they stabilize the rigid helical structure.

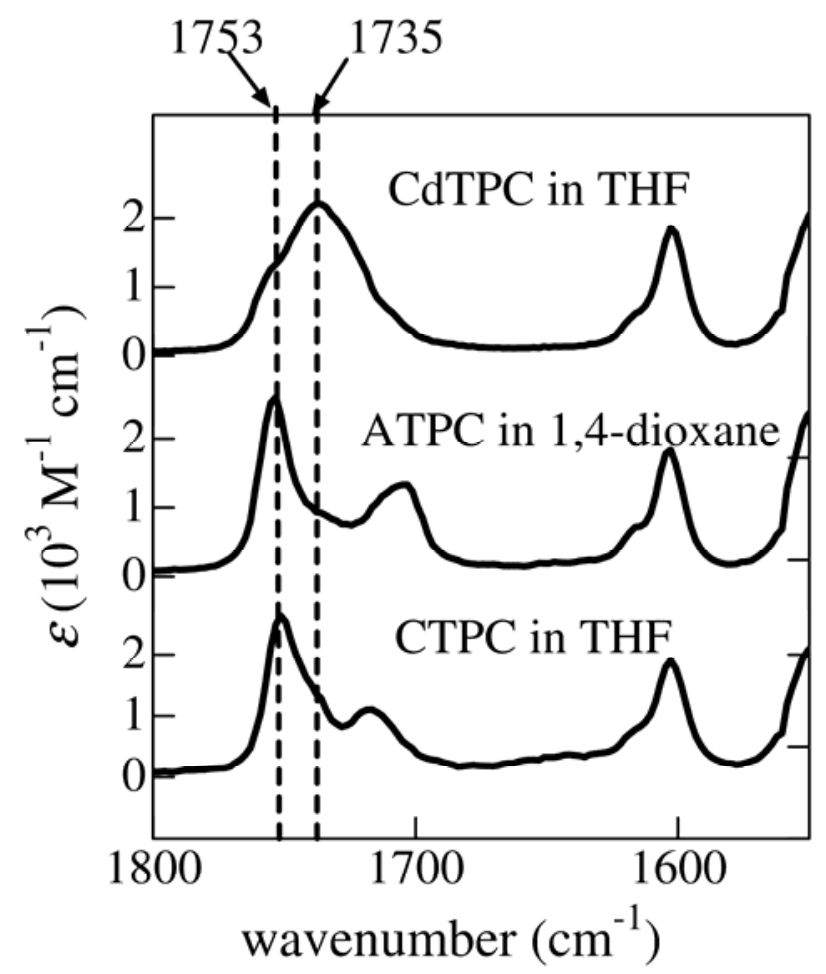

Fig. 6. IR spectra (molar absorption coefficient $\varepsilon$ vs wavenumber) for CdTPC1500K in THF, for an ATPC sample in 1,4-dioxane $\left(M_{\mathrm{w}}=3.3 \times 10^{6}\right)$ [5] and for CTPC in THF $\left(M_{\mathrm{w}}=2.6 \times\right.$ $10^{6}$ ) at $25^{\circ} \mathrm{C}$ from top to the bottom.

\section{Concluding Remarks}

While single-chain curdlan behaves as rather flexible chain in solution, its phenylcarbamate derivative (CdTPC) has much higher chain stiffness (57 nm in the Kuhn segment length $\lambda^{-1}$ ) in THF and the helix pitch per residue is substantially the same as those for single helical curdlan and curdlan triacetate in the crystalline state. Considering the chain stiffness of CdTPC becomes much smaller $\left(\lambda^{-1}=17 \mathrm{~nm}\right)$ in MIBK, we may conclude that 
CdTPC has the tightly wound single helical structure in THF. This chain stiffness is also much higher than those for amylose and cellulose tris(phenylcarbamate)s and close to the highest value (75 nm) [7] in single helical polysaccharides and their derivatives.

\section{Acknowledgments}

The synchrotron radiation experiments were performed at the BL40B2 in SPring-8 with the approval of the Japan Synchrotron Radiation Research Institute (JASRI) (Proposal \#2008A1313 and \#2009A1049). This work was partially supported by Grant-in-Aid for Young Scientists (\#23750128) from Japan Society for the Promotion of Science (JSPS).

\section{References}

[1] Burchard W. Light scattering from polysaccharides as soft materials. In: Borsali R, Pecora R, editors. Soft-matter characterization, vol. 1. Springer, 2008. pp. 463-603.

[2] Bittiger H, Keilich G. Biopolymers 1969;7:539-556.

[3] Nakanishi Y, Norisuye T, Teramoto A, Kitamura S. Macromolecules 1993;26:4220-5.

[4] Kratky O, Porod G. Recl Trav Chim Pays-Bas 1949;68:1106-22.

[5] Terao K, Fujii T, Tsuda M, Kitamura S, Norisuye T. Polym J 2009;41:201-7.

[6] Kasat RB, Zvinevich Y, Hillhouse HW, Thomson KT, Wang NHL, Franses EI. J. Phys. Chem. B. 2006;110:14114-14122.

[7] Terao K, Murashima M, Sano Y, Arakawa S, Kitamura S, Norisuye T. Macromolecules 2010;43:1061-1068.

[8] Sato, T.; Shimizu, T.; Kasabo, F.; Teramoto, A. Macromolecules 2003, 36, 2939-2943.

[9] Kuse, Y.; Asahina, D.; Nishio, Y. Biomacromolecules 2009, 10, 166-173.

[10] Harada T, Misaki A, Saito H. Arch Biochem Biophys 1968;124:292-8.

[11] Miyoshi K, Uezu K, Sakurai K, Shinkai S. Chem Biodivers 2004;1:916-24.

[12] Sano Y, Terao K, Arakawa S, Ohtoh M, Kitamura S, Norisuye T. Polymer 2010;51:4243-8.

[13] Fujii T, Terao K, Tsuda M, Kitamura S, Norisuye T. Biopolymers 2009;91:729-36.

[14] Kasabo F, Kanematsu T, Nakagawa T, Sato T, Teramoto A. Macromolecules 2000;33:2748-56. 
[15] Okamoto Y, Noguchi J, Yashima E. React Func Polym 1998;37:183-8.

[16] Berry GC. J Chem Phys 1966;44:4550-64.

[17] Nakata M, Kawaguchi T, Kodama Y, Konno A. Polymer 1998;39:1475-81.

[18] Hirano I, Einaga Y, Fujita H. Polym J 1979;11:901-4.

[19] Benoit H, Doty P. J Phys Chem 1953;57:958-63.

[20] Domb C, Barrett AJ. Polymer 1976;17:179-84.

[21] Yamakawa H. Helical Wormlike Chains in Polymer Solutions. Berlin: Springer, 1997.

[22] Yamakawa H, Stockmayer WH. J Chem Phys 1972;57:2843-54.

[23] Shimada J, Yamakawa H. J. Chem Phys 1986;85:591-9.

[24] Nakamura Y, Norisuye T. J Polym Sci Part B Polym Phys 2004;42:1398-407.

[25] Konishi T, Yoshizaki T, Saito T, Einaga Y, Yamakawa H. Macromolecules 1990;23:290-7.

[26] Yamakawa H, Fujii M. Macromolecules 1974;7:128-35.

[27] Yamakawa H, Yoshizaki T. Macromolecules 1980;13:633-43.

[28] Barrett AJ. Macromolecules 1984;17:1566-72.

[29] Tsuda T, Terao K, Nakamura Y, Kita Y, Kitamura S, Sato T. Macromolecules 2010;43:5779-84.

[30] Okuyama K, Otsubo A, Fukuzawa Y, Ozawa M, Harada T, Kasai N. J Carbohyd Chem 1991;10:645-56.

[31] Okuyama K, Obata Y, Noguchi K, Kusaka T, Ito Y, Ohno S. Biopolymers 1996;38:55766.

[32] Tsuda T, Terao K, Kitamura S, Sato T. submitted. 